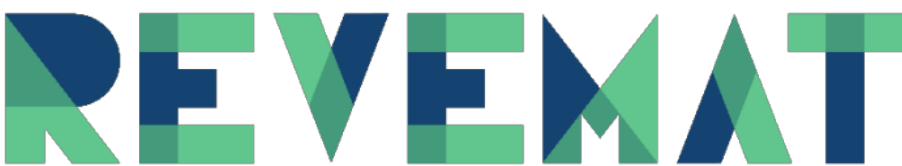

REVISTA ELETRÔNICA DE EDUCAÇÃO MATEMÁTICA

\title{
OS TRÊS MUNDOS DA MATEMÁTICA NA FORMAÇÃO DE PROFESSORES QUE ENSINAM MATEMÁTICA: O CÁLCULO DA ÁREA LIMITADA POR REGIÕES POLIGONAIS REGULARES
}

\section{The Three Mathematics Worlds in the education of teachers that teach Mathematics: the calculation of the area limited by regular polygonal regions}

\author{
Geraldo Aparecido POLEGATTI \\ Instituto Federal de Mato Grosso, Juína, Mato Grosso, Brasil. \\ geappolegatti@gmail.com \\ Ohttps://orcid.org/0000-0003-4515-3855
}

Angela Marta Pereira das Dores SAVIOLI Universidade Estadual de Londrina, Londrina, Paraná, Brasil. angelamarta@uel.br

Ohttps://orcid.org/0000-0002-5624-6398

A lista completa com informações dos autores está no final do artigo

\begin{abstract}
RESUMO
Neste artigo tem-se o objetivo de discutir o estudo dos Três Mundos da Matemática (Corporificado, Simbólico e Formal) de David Tall, no processo de formação inicial ou continuada de professores que ensinam Matemática. Ele desponta-se como um quadro teórico amplo, articulado e dinâmico que esquematiza o conhecimento matemático desenvolvendo-se em longo prazo por intermédio das ações humanas (professores e estudantes) nos objetos matemáticos. Englobam-se três formas matemáticas (prática, teórica e formal) de conduzir o pensamento matemático no transcorrer de processos de ensino e de aprendizagem da Matemática que se inter-relacionam com três maneiras de abstrair-se matematicamente (estrutural, operacional e formal). Dialoga-se uma situação hipotética com o cálculo da área de regiões limitadas por polígonos regulares em função do raio da circunferência que o circunscreve e tendo como base a área da região de um triângulo qualquer, a partir do valor do seno de um de seus ângulos internos. Trata-se de uma pesquisa de cunho bibliográfico. Ao final estendem-se as discussões para o cálculo da área da região limitada por um círculo.
\end{abstract}

Palavras-chave: Educação Matemática, Três Mundos da Matemática, Polígonos Regulares

\begin{abstract}
This article aims to discuss the study of the Three Worlds of Mathematics (Embodied, Symbolic and Formal) by David Tall, in the process of initial or continuing education of teachers who teach Mathematics. It emerges as a broad, articulate and dynamic theoretical framework that outlines mathematical knowledge developing in the long term through human actions (teachers and students) in mathematical objects. Embrace three mathematical forms (practical, theoretical and formal) of conducting mathematical thinking in the course of teaching and learning processes in Mathematics are included, which are interrelated with three ways of mathematically abstracting (structural, operational and formal). A hypothetical situation is discussed with the calculation of the area of regions bounded by regular polygons
\end{abstract}


as a function of the radius of the circumference that circumscribes it and based on the area of the region of any triangle, from the sine value of one of its angles internal. This is a bibliographic research. At the end, the discussions for the calculation of the area of the region limited by a circle are extended.

Keywords: Mathematical Education, Three Worlds of Mathematics, Regular Polygons

\section{INTRODUÇÃO}

Neste artigo tem-se o objetivo de discutir a implementação de estudos envolvendo os Três Mundos da Matemática de David Tall no campo da Educação Matemática. Para tanto, fomenta-se uma proposta de ensino e de aprendizagem que envolve um caso hipotético com o cálculo das áreas de regiões limitadas por polígonos regulares quaisquer, em função do raio da circunferência que os circunscreve e, tendo como base, a fórmula que se utiliza para calcular a área da região limitada por um triângulo qualquer, a partir do valor do seno de um de seus ângulos internos. Trata-se de uma pesquisa bibliográfica direcionada a formação de professores que ensinam Matemática sob a luz do quadro teórico dos Três Mundos da Matemática.

Para Tall (2013), o conhecimento matemático se desenvolve em três mundos matemáticos que se inter-relacionam formando uma estrutura coesa e dinâmica com diferenciados graus de aprimoramento do pensamento matemático. $\mathrm{O}$ Mundo Corporificado focaliza as características tanto físicas quanto conceituais (construídas mentalmente) dos objetos matemáticos. O Mundo Simbólico contempla as operações matemáticas por intermédio de manipulações simbólicas e suas propriedades. O Mundo Formal trabalha as definições presentes no conhecimento matemático, em função de teoremas e linguagem axiomática utilizando a teoria dos conjuntos e a lógica matemática.

De acordo com Bisognin; Bisognin \& Leivas (2016), em Os Três Mundos da Matemática o professor formador tem a possibilidade de planejar, executar e analisar os resultados de práticas educacionais, no processo de formação de professores que ensinam Matemática, bem como discutir com os acadêmicos a sua utilização no transcorrer das atividades. Ele é um quadro abrangente que apresenta o conhecimento matemático se aprimorando em três vertentes do pensamento matemático (matemática prática, matemática teórica e matemática formal) e, em três níveis de abstração (estrutural, operacional e formal). Para os autores as "oportunidades de ir e vir entre os Três Mundos da Matemática devem ser estimuladas pelos docentes formadores, em qualquer conteúdo trabalhado nas disciplinas" (p. 374). 
No caso hipotético proposto, as discussões fluem envolvendo o cálculo da área de pentágonos e octógonos regulares, a partir da área de qualquer triângulo por meio do seno de um de seus ângulos internos. O quadro teórico dos Três Mundos da Matemática permeia os processos de ensino e de aprendizagem propostos pelo professor formador. Ao final alongam-se as discussões com o cálculo da área da região limitada por um círculo qualquer, por meio da fórmula apresentada para o cálculo da área da região limitada por um polígono regular. A seguir faz-se uma breve apresentação sobre os Três Mundos da Matemática.

\section{PRELIMINARES ACERCA DOS TRÊS MUNDOS DA MATEMÁTICA}

Segundo Schastai (2017), o quadro teórico dos Três Mundos da Matemática atua como um mapa que aponta possíveis caminhos acerca do desenvolvimento do pensamento matemático em longo prazo, desde o pensamento mais elementar ao mais avançado. Esse quadro teórico possibilita o desdobramento de jornadas cognitivas de estudantes e professores envolvidos em algum processo de ensino e de aprendizagem da Matemática. A socialização dessas percepções e a comunicação delas por meio de uma linguagem simbólica instigam o aprimoramento das categorizações iniciais, incentiva a construção, a descrição e a definição de outras propriedades que podem ser utilizadas para a prova matemática.

De acordo com Tall (2008), o conhecimento matemático se desenvolve em Três Mundos da Matemática (Corporificado, Simbólico e Formal) de forma diferenciada entre eles, mas que se inter-relacionam formando uma tríade coesa e articulada, que envolve ações (observar, medir, manusear, contar, ordenar, classificar, modelar, inferir, demonstrar, entre outras) humanas em objetos matemáticos reais ou imaginados. Para o autor, o Mundo Corporificado se constitui por meio da percepção inicial em objetos matemáticos reias, cujas ações desenvolvidas pelo ser humano conduzem a reflexões sobre suas propriedades, e estas, são construídas pelo indivíduo em sua mente transformando os objetos reais, doravante perseptíveis, em objetos matemáticos construídos em sua mente. "Nesse mundo, as propriedades de um objeto são observadas e compreendidas" (Lima, 2019, p. 8).

No âmbito do Mundo Simbólico as ações humanas nos objetos matemáticos promovem manipulações simbólicas. "Esses símbolos podem ser vistos de maneira 
flexível tanto como o procedimento a ser efetuado, quanto como o resultado obtido a partir do desenvolvimento desse procedimento, que é o conceito" (Lima, 2019, p. 8). Tall (2008) salienta que o Mundo Simbólico se desenvolve a partir do Mundo Corporificado por intermédio das ações nos objetos matemáticos, que se constroem na mente do ser humano, englobando manipulaçãoes e operaçãoes matemáticas, numéricas ou algébricas, incentiva-se a articulação entre os conceitos que compõem o pensamento matemático e os processos que dizem respeito aos modos de como se proceder ou fazer determinada manipulação simbólica ou operação matemática.

Com relação ao Mundo Formal, Tall (2008) nos informa que ele se contitui com base em objetos matemáticos conhecidos, transformando-os em objetos formais, por meio de linguagem matemática organizada em axiomas e teoremas que conduzem à prova matemática, tendo como base, a teoria dos conjuntos e a lógica matemática. No interior do Mundo Formal "axiomas, propriedades, definições e teoremas são usados para construir o corpo axiomático da Matemática" (Lima, 2019, p. 8). Apesar de não se trabalhar a linguagem axiomática nas séries iniciais do Ensino Fundamental, em qualquer atividade matemática há presença de características do Mundo Formal que são fundamentais para a construção de conceitos matemáticos (Lima, 2019).

Bueno \& Viali (2019), destacam que cada um dos Três Mundos da Matemática possui características diferenciadas que os delimitam, mas intersecções entre os mundos são inerentes quando vistos como um quadro teórico que compreende e abarca o conhecimento matemático. Da mesma maneira, Visintainer (2019), ressalta que para se executar demonstrações matemáticas presentes no Mundo Formal utilizam-se conceitos e processos característicos do Mundo Simbólico e até percepções com ações em objetos matemáticos que se constituem no âmbito do Mundo Corporificado.

Tall (2013) salienta que o ápice de aprimoramento do pensamento matemático se concretiza com o entendimento e realização da prova matemática, mas essa compreensão repercute tanto no Mundo Simbólico, justificando manipulações simbólicas que sem essa prova matemática não seriam prováveis, quanto no Mundo Corporificado com o desenvolvimento de novas corporificações que conduzem a outras reflexões, e estas, incidem na composição de mais simbolismos matemáticos, e estes precisam ser demonstrados por meio de prova matemática.

Em seguida apresenta-se o quadro teórico dos Três Mundos da Matemática articulando-se os movimentos horizontais do pensamento matemático, em função dos três modos distintos de se agir matematicamente (matemática prática, matemática teórica e 
matemática formal), com os movimentos verticais do pensamento matemático, em função das três etapas de desenvolvimento cognitivo (abstração estrutural, abstração operacional e abstração formal), em um panorama geral que sintetiza o conhecimento matemático desenvolvendo-se em longo prazo.

\section{A DINÂMICA DO QUADRO TEÓRICO DOS TRÊS MUNDOS DA MATEMÁTICA}

Segundo Tall (2019), a matemática prática diz respeito às ações advindas da percepção humana em objetos inicialmente reais e, posteriormente, essas ações atuam de forma simultânea conduzindo à construção de propriedades de objetos matemáticos mentais que se articulam de maneiras coerentes. No âmbito escolar, as ações envolvendo matemática prática são aprimoradas com base em deduções e definições com vistas a aplicações da Matemática.

Conforme Tall (2019), a matemática teórica relaciona as ideias e manipulações simbólicas entre si, utilizando deduções e definições que são construídas a partir das percepções humanas em objetos matemáticos, e estes, são cada vez mais aprimorados na mente do indivíduo. Já a matemática formal é baseada em axiomas e teoremas para a construção de propriedades formais, que conduzem à prova matemática, para satisfazer esses mesmos axiomas e teoremas, utiliza-se para tanto, a teoria dos conjuntos e a lógica matemática (Tall, 2019). Para Tall (2020), a prova formal conduz a formas aprimoradas de corporificações e simbolismos englobando em uma estrutura dinâmica os Três Mundos da Matemática (Corporificado, Simbólico e Formal) em três níveis de sofisticação das ações do pensamento matemático (prática, teórica e formal).

De acordo com Tall (2013), a abstração estrutural desenvolve-se por intermédio das ações do ser humano em objetos reais cujas propriedades matemáticas são desencadeadas no imaginário de cada indivíduo. Então, a partir de objetos perceptíveis aos sentidos (observação e manuseio) eles são identificados e classificados segundo suas propriedades matemáticas. A abstração operacional desenvolve-se por meio das ações humanas com objetos matemáticos (expressões, equações, funções, entre outras), envolvendo operações aritméticas e ou algébricas buscando intensificar a manipulação simbólica. Já a abstração formal desenvolve-se mediante as ações do ser humano com operações formais da Matemática (definições, axiomas, teoremas, entre outras) para a 
construção de novas propriedades formais e o desdobramento de prova matemática.

Segundo Schastai (2017), a abstração formal representa um salto significativo no desenvolvimento do conhecimento matemático por parte do ser humano, pois essa etapa de aprimoramento cognitivo é constituída, essencialmente, por definições oriundas da linguagem axiomática, mas que mantém vínculos com as percepções e as manipulações simbólicas presentes nas abstrações estrutural e operacional, respectivamente. "Inconscientemente, pode continuar a ter ligações com percepção e ação, mas formalmente, oferece uma nova abordagem, universal para a matemática em que os teoremas provados dependem somente de definição e de demonstração" (Schastai, 2017, p. 55). Assim, apresenta-se na Figura 1 o quadro teórico dos Três Mundos da Matemática que emerge como um guia que traz possibilidades para o desenvolvimento de jornadas cognitivas de estudantes e professores no planejamento, execução e posteriores análises de processos de ensino e de aprendizagem da Matemática.

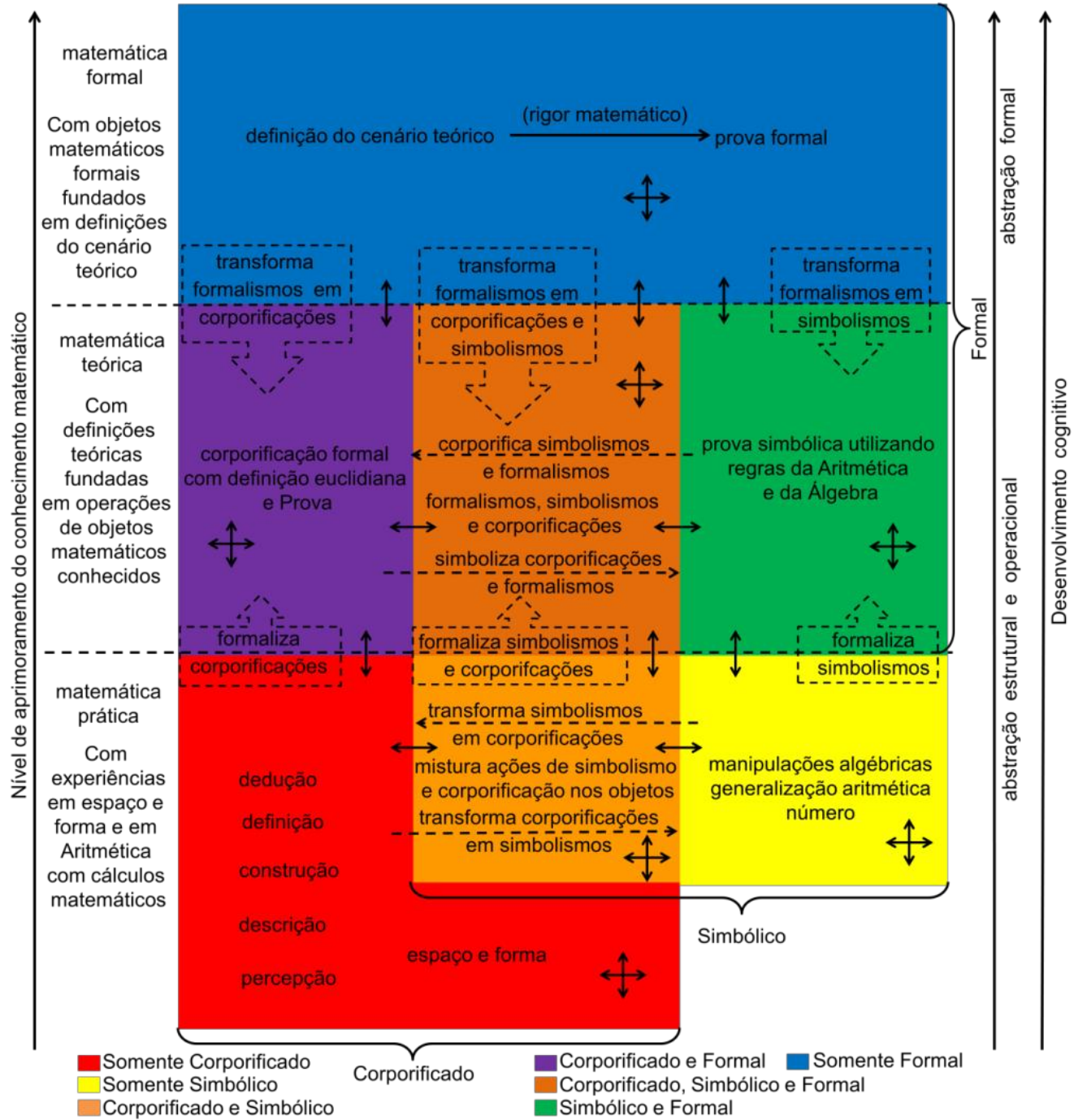

Figura 1: O quadro teórico dos Três Mundos da Matemática

Fonte: Elaborado por Polegatti (2020) com base em Tall (2007; 2008; 2013; 2019; 2020) 
Doravante, para evitar repetições nas descrições utilizam-se siglas com as iniciais maiúsculas de cada mundo. Assim, as áreas das regiões limitadas com as cores primárias vermelha, amarela e azul indicam, respectivamente, os espaços com características exclusivas dos mundos Corporificado (C), Simbólico (S) e Formal (F). Assim como indica Polegatti (2020), as áreas das regiões limitadas com as cores secundárias, verde (amarela + azul), laranja clara (amarela + vermelha) e roxa (vermelha + azul) representam, na mesma sequência, as zonas de confluências entre os mundos Corporificado e Simbólico (CS), Corporificado e Formal (CF), Simbólico e Formal (SF). E a área da região limitada pela cor terciária laranja escura (vermelho + azul + amarela), indica a zona de confluência entre os Três Mundos da Matemática Corporificado, Simbólico e Formal (CSF).

Tall (2019) salienta que dependendo do contexto de formação de cada indivíduo que utilize o quadro teórico dos Três Mundos da Matemática, inevitavelmente, ele pode ser interpretado por diferentes maneiras pessoais de pensar e de dar significados ao desenvolvimento do conhecimento matemático.

Minha visão é construída como resultado de uma jornada pessoal em que estudei um doutorado em matemática pura com Michael Atiyah, que recebeu uma medalha Fields na época e, posteriormente, um doutorado em psicologia do ensino de matemática com Richard Skemp, que era um líder mundial em educação matemática. Isso me permite dotar o diagrama de uma rica variedade de significados. No entanto, um leitor, com experiências e desenvolvimento completamente diferentes, pode ler o diagrama de várias outras maneiras (p. 14, tradução nossa $\left.{ }^{1}\right)$.

Tendo como cenário o quadro teórico da Figura 1, esquematiza-se a dinâmica de movimento do pensamento matemático, no transcorrer de quaisquer processos de ensino e de aprendizagem da Matemática e, em qualquer nível escolar, ao combinarem-se suas transições de idas e vindas (horizontais), por meio das ações de matemáticas prática, teórica e formal, com as transições de subidas e descidas por intermédio do desenvolvimento das abstrações estrutural, operacional e formal. O símbolo $(\hat{\leftrightarrow})$ representa o deslocamento do pensamento matemático em cada mundo ou em suas zonas de confluência conciliando ações matemáticas com os desdobramentos de abstrações (Polegatti, 2020).

\footnotetext{
1 "My own view is built as the result of a personal journey in which I studied for a doctorate in pure mathematics with Michael Atiyah, who was awarded a Fields medal at the time, and later for a doctorate in the psychology of mathematics education with Richard Skemp, who was a world leader in mathematics education. This allows me to endow the diagram with a rich array of meanings. However, a reader, with completely different experiences and development, may read the diagram in many other ways".
} 
Por sua vez, seguindo o quadro teórico da Figura 1, para Polegatti (2020), o sinal $(\leftrightarrow)$ indica as travessias horizontais das fronteiras entre os mundos ou zonas de confluências desencadeadas pela dinâmica de movimento do pensamento matemático, em cada um dos três níveis de aprimoramento das ações do ser humano nos objetos matemáticos (matemática prática, matemática teórica e matemática formal). Já o símbolo $(\mathfrak{l})$ diz respeito aos deslocamentos verticais do pensamento matemático, que atravessam as fronteiras entre os mundos e suas zonas de confluências, promovendo transições entre as matemáticas prática, teórica e formal, bem como o possível aprimoramento cognitivo do viajante no desenvolvimento das abstrações estrutural, operacional e formal (Polegatti, 2020).

Ressalta-se que a dinâmica de movimento do pensamento matemático não acontece de forma linear, ora só horizontal, ora só vertical. Esse movimento é dinâmico, complexo, flexível, espontâneo ou provocado, estimulado, com idas e vindas, subidas e descidas, que se desenvolve em longo prazo. O Quadro 1, em acordo com Polegatti (2020), sintetiza a dinâmica de movimento do pensamento matemático nos Três Mundos da Matemática.

Quadro 1: A dinâmica de movimento do pensamento matemático nos Três Mundos da Matemática

\begin{tabular}{|c|c|}
\hline Transição & Direção: Vertical (V) elou Horizontal (H) \\
\hline$C \stackrel{\leftrightarrow}{\hookrightarrow} C$ & $\begin{array}{l}\text { H: ações de matemática prática restritas ao Mundo Corporificado. Envolvem-se } \\
\text { corporificações com ou sem indícios de simbolismos. O pensamento matemático ocorre } \\
\text { por meio da percepção com destaque para a observação, a experimentação, bem como } \\
\text { a descrição de figuras e sólidos geométricos. Podem-se formar conjuntos com essas } \\
\text { figuras e sólidos e envolvem-se as noções de: espaço, gráficos, arcos e ângulos. } \\
\text { V: desenvolvimento de abstração estrutural com ou sem indícios de abstração } \\
\text { operacional no âmbito do Mundo Corporificado. Provoca-se o aprimoramento da } \\
\text { linguagem pictórica (por meio de figuras, desenhos, pictogramas) processo que tem } \\
\text { início na construção, passa-se pela definição, chega-se à dedução informal e a análise } \\
\text { de objetos matemáticos, principalmente, as figuras e os sólidos geométricos, os gráficos } \\
\text { (pictogramas), ângulos, arcos, a construção geométrica das cônicas (parábola, elipse e } \\
\text { hipérbole) a partir das secções de um cone, ambos sem envolver a ideia de número. }\end{array}$ \\
\hline $\mathrm{C} \leftrightarrow \mathrm{CS}$ & $\begin{array}{l}\text { H: ações de matemática prática para transposição de fronteira entre o Mundo } \\
\text { Corporificado e a zona de confluência dos Mundos Corporificado e Simbólico. Dentre } \\
\text { elas, introduz-se a ideia de número (Naturais e Inteiros) associada, por exemplo, às } \\
\text { figuras e aos sólidos geométricos, tabelas, gráficos, ângulos e arcos. A compreensão e } \\
\text { adoção de um símbolo para o número zero possibilitam o estudo e a utilização do } \\
\text { sistema decimal posicional hindu-arábico. }\end{array}$ \\
\hline $\mathrm{CS} \stackrel{\leftrightarrow}{\leftrightarrow} \mathrm{CS}$ & $\begin{array}{l}\text { H: ações de matemática prática no interior da zona de confluência entre o Mundo } \\
\text { Corporificado e o Mundo Simbólico para simbolizar corporificações ou corporificar } \\
\text { simbolismos. Por exemplo, a resolução de equações (equacionar = equilibrar) por meio } \\
\text { do dispositivo conhecido como balança de dois pratos. Não há somente a observação e } \\
\text { manipulação de figuras, sólidos geométricos e gráficos, mas as suas dimensões são } \\
\text { notadas e mensuradas pelo indivíduo. } \\
\text { V: desenvolvimento de abstração estrutural e ou abstração operacional no âmbito dessa } \\
\text { zona de confluência. O aprimoramento do pensamento matemático emerge, por } \\
\text { exemplo, no estudo dos conjuntos numéricos, com a construção e interpretação de }\end{array}$ \\
\hline
\end{tabular}




\begin{tabular}{|c|c|}
\hline & $\begin{array}{l}\text { tabelas numéricas, na aferição de comprimentos, alturas, larguras, perímetros, ângulos, } \\
\text { arcos, ao resolverem-se as quatro operações fundamentais, a potenciação com } \\
\text { expoentes naturais, a radiciação com seu índice sendo um número Natural, nas } \\
\text { resoluções de expressões numéricas envolvendo as operações matemáticas } \\
\text { (manipulações aritméticas podendo envolver as figuras e os sólidos geométricos), com a } \\
\text { resolução dos problemas de contagem por meio de fatorial e, com o estudo dos } \\
\text { logaritmos aprimorando as técnicas de cálculos em Aritmética. }\end{array}$ \\
\hline $\mathrm{CS} \leftrightarrow \mathrm{S}$ & $\begin{array}{l}\mathrm{H} \text { : ações de matemática prática para transposição de fronteira entre a zona de } \\
\text { confluência dos Mundos Corporificado e Simbólico para o Mundo Simbólico. A } \\
\text { introdução de letras como coeficientes e a invenção dos números complexos são } \\
\text { possíveis divisores nesse deslocamento. No aprimoramento do pensamento matemático } \\
\text { a abstração operacional se sobressai perante a abstração estrutural, nas ações de } \\
\text { matemática prática para a construção do conceito de incógnita, que compõe a ideia de } \\
\text { associar uma letra do alfabeto a um valor numérico. }\end{array}$ \\
\hline$S \leftrightarrow s$ & $\begin{array}{l}\text { H: aprimora-se o pensamento matemático por meio de ações de matemática prática } \\
\text { envolvendo simbolismos com ou sem indícios de corporificações, ambas restritas ao } \\
\text { Mundo Simbólico. Dentre elas, promove-se o cálculo de áreas e volumes das figuras e } \\
\text { dos sólidos geométricos, respectivamente, e introduz-se a ideia de variável (associação } \\
\text { de uma letra do alfabeto a valores numéricos variáveis). } \\
\text { V: desenvolve-se abstração operacional com ou sem indícios de abstração estrutural no } \\
\text { cenário do Mundo Simbólico. Por exemplo, os conceitos de incógnita e variável são } \\
\text { vinculados às figuras e aos sólidos geométricos, gráficos e tabelas. Englobam-se os } \\
\text { cálculos de comprimentos, áreas e volumes além da resolução de equações envolvendo } \\
\text { esses cálculos, bem como o aprimoramento dos conceitos de incógnita com a resolução } \\
\text { de sistemas de equações e de variável com a identificação e construção da ideia de } \\
\text { função matemática. }\end{array}$ \\
\hline$C \uparrow C F$ & $\begin{array}{l}\text { V: transposição entre o Mundo Corporificado e a zona de confluência entre o Mundo } \\
\text { Corporificado e o Mundo Formal. Desenvolve-se abstração estrutural com ou sem } \\
\text { indícios de abstração operacional e, com vistas a indícios de abstração formal. } \\
\text { Aprimora-se o pensamento matemático com a transição da matemática prática para a } \\
\text { matemática teórica por intermédio de ações para formalizar corporificações. Dentre elas, } \\
\text { destacam-se as técnicas de desenho geométrico com régua e compasso no âmbito da } \\
\text { Geometria Euclidiana indo da dedução informal para a dedução formal. }\end{array}$ \\
\hline 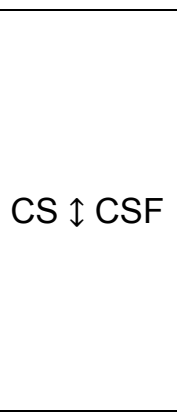 & $\begin{array}{l}\text { V: transposição entre a zona de confluência dos mundos Corporificado e Simbólico e a } \\
\text { zona de confluência que apresenta características dos Três Mundos da Matemática } \\
\text { (Corporificado Simbólico Formal). Desenvolve-se abstração estrutural e ou abstração } \\
\text { operacional e, com vistas a indícios de abstração formal. Aprimora-se o pensamento } \\
\text { matemático com a transição da matemática prática para a matemática teórica por meio } \\
\text { de ações para formalizar corporificações e simbolismos. Por exemplo, o cálculo de } \\
\text { potenciação com expoentes e de radiação com índices inteiros e, posteriormente, } \\
\text { racionais. Introduz-se a segunda incógnita nas equações (sistema de equações), } \\
\text { estudos e discussões sobre os infinitesimais e o princípio fundamental da contagem } \\
\text { com a necessidade posterior dele ser demonstrado teoricamente e formalmente. }\end{array}$ \\
\hline$S \uparrow S F$ & $\begin{array}{l}\mathrm{V} \text { : transposição entre o Mundo Simbólico e a zona de confluência dos mundos } \\
\text { Simbólico e Formal. Desenvolve-se abstração operacional com ou sem indícios de } \\
\text { abstração estrutural e, com vistas a indícios de abstração formal. Aprimora-se o } \\
\text { pensamento matemático com a transição da matemática prática para a matemática } \\
\text { teórica por meio de ações para formalizar simbolismos. Por exemplo, o desenvolvimento } \\
\text { teórico do conceito de função matemática que até antes dessa transição fora utilizado } \\
\text { em situações práticas. }\end{array}$ \\
\hline $\mathrm{CF} \stackrel{\leftrightarrow}{\leftrightarrow} \mathrm{CF}$ & $\begin{array}{l}\text { H: ações de matemática teórica no interior da zona de confluência entre os mundos } \\
\text { Corporificado e Formal. Envolve-se formalização de corporificações ou corporificação de } \\
\text { formalismos com ou sem indícios de simbolismos. Por exemplo, estudos das } \\
\text { características formais das figuras e dos sólidos geométricos, a prova euclidiana é } \\
\text { sinônima desse processo. } \\
\text { V: desenvolve-se abstração estrutural com indícios da abstração formal e, com ou sem } \\
\text { indícios da abstração operacional. Intensifica-se o aprimoramento do pensamento } \\
\text { matemático por meio da prova geométrica como, por exemplo, estudos dos princípios } \\
\text { de Arquimedes e Cavalieri, do Método da Exaustão e do Teorema de Pitágoras } \\
\text { utilizando desenhos geométricos por meio da comparação de áreas (demonstração }\end{array}$ \\
\hline
\end{tabular}




\begin{tabular}{|c|c|}
\hline & $\begin{array}{l}\text { geométrica). Promove-se discussões sobre os entes geométricos ponto, reta, segmento } \\
\text { de reta, plano, envolve-se demonstrações geométricas por meio das técnicas de } \\
\text { desenho geométrico utilizando régua e compasso, bem como se inclui estudos das } \\
\text { geometrias não euclidianas expandindo o escopo de estudos da Geometria. }\end{array}$ \\
\hline $\mathrm{CF} \leftrightarrow \mathrm{CSF}$ & $\begin{array}{l}\text { H: ações de matemática teórica para transposição da fronteira entre a zona de } \\
\text { confluência dos mundos Corporificado e Formal com a zona de tripla interseç̧ão dos } \\
\text { Três Mundos da Matemática. Dentre elas, destaca-se o aprimoramento do pensamento } \\
\text { matemático com a associação entre a Geometria e a Álgebra, da qual emerge a } \\
\text { Geometria Analítica. }\end{array}$ \\
\hline $\mathrm{CSF} \leftrightarrow \mathrm{CSF}$ & $\begin{array}{l}\text { H: ações de matemática teórica no interior da zona de tripla confluência entre os Três } \\
\text { Mundos da Matemática com vistas a simbolizar corporificações e formalismos e ou } \\
\text { corporificar simbolismos e formalismos. Aprimora-se o pensamento matemático com } \\
\text { elementos tanto da Geometria quanto da Álgebra e da Aritmética. Envolvem-se } \\
\text { corporificações nas resoluções de aplicações matemáticas. } \\
\text { V: desenvolvem-se de abstrações estrutural e operacional com indícios de abstração } \\
\text { formal. As técnicas de prova geométrica são entrelaçadas às técnicas de prova } \\
\text { algébrica. Promovem-se discussões dos procedimentos de integração (cálculo de áreas, } \\
\text { integral definida, função área) e derivação como taxas de variação, estudos com a ideia } \\
\text { de limite e as somas de Riemann emergem nas discussões. Intensifica-se o debate } \\
\text { acerca da Teoria dos Conjuntos. A resolução de sistemas de equações ocorre tanto } \\
\text { geometricamente (intersecção de retas ou curvas), quando algebricamente (cálculo do } \\
\text { conjunto solução). A Geometria Analítica se desenvolve, por exemplo, com o estudo dos } \\
\text { elementos geométricos como retas, planos, circunferências, parábolas, elipses, } \\
\text { hipérboles, que são associadas a suas equações algébricas e paramétricas e que } \\
\text { possibilitam manipulações algébricas e geométricas com aportes da Aritmética. } \\
\text { Transformam-se os variados tipos de equações em funções matemática, e essas, são } \\
\text { incorporadas às manipulações simbólicas conduzindo ao aprimoramento do } \\
\text { pensamento matemático com relação aos conceitos de derivação por meio da inclinação } \\
\text { da reta tangente e integração com o cálculo de integrais indefinidas. }\end{array}$ \\
\hline $\mathrm{CSF} \leftrightarrow \mathrm{SF}$ & $\begin{array}{l}\text { H: ações de matemática teórica para transposição de fronteira entre a zona de tripla } \\
\text { confluência dos Três Mundos da Matemática e a zona de intersecção entre os mundos } \\
\text { Simbólico e Formal. Aprimora-se o pensamento matemático ao Intensificar-se a } \\
\text { dedução, por meio da prova simbólica, de elementos da Geometria Analítica com } \\
\text { conceitos da Álgebra Linear. Por exemplo, com os cálculos das aplicações de limites, } \\
\text { derivadas e integrais de funções matemáticas, por meio de manipulações algébricas nas } \\
\text { resoluções de problemas com posterior prova algébrica. }\end{array}$ \\
\hline$S F \stackrel{\leftrightarrow}{\leftrightarrow} S F$ & $\begin{array}{l}\text { H: ações de matemática teórica restritas à zona de confluência entre os mundos } \\
\text { Simbólico e Formal. Englobam-se simbolismos com indícios de formalismos, com ou } \\
\text { sem indícios de corporificações. É nesse cenário que se tem o início da formalização da } \\
\text { Geometria Analítica e da Álgebra Linear. } \\
\text { V: desenvolve-se abstração operacional com indícios de abstração formal, com ou sem } \\
\text { indícios de abstração estrutural. Aprimora-se o pensamento matemático ao Intensificar- } \\
\text { se a manipulação algébrica por meio de mecanismos ou técnicas de demonstrações e } \\
\text { prova algébrica, preparando o escopo teórico para a implementação do Sistema } \\
\text { Axiomático Formal. }\end{array}$ \\
\hline$C F \uparrow F$ & $\begin{array}{l}\text { V: transposição entre a zona de confluência dos mundos Corporificado e Formal e o } \\
\text { Mundo Formal. Desenvolve-se abstração estrutural com vistas à abstração formal, com } \\
\text { ou sem indícios de abstração operacional. Aprimora-se o pensamento matemático com } \\
\text { a transição da matemática teórica para a matemática formal. Proporcionam-se } \\
\text { discussões fomentadas por alguns matemáticos como: Eudoxo, Arquimedes, Tales, } \\
\text { Pitágoras, Euclides, entre outros. }\end{array}$ \\
\hline CSF $\uparrow F$ & $\begin{array}{l}\text { V: transposição entre a zona de tripla confluência dos Três Mundos da Matemática e o } \\
\text { Mundo Formal. Desenvolvem-se e abstrações estrutural e operacional com vistas à } \\
\text { abstração formal. Ocorre a transição da matemática teórica para a matemática formal. } \\
\text { Provoca-se aprimoramento do pensamento matemático com a imersão nos estudos das } \\
\text { deduções e demonstrações em teoremas com destaque para o Teorema Fundamental } \\
\text { do Cálculo. Abre-se espaço teórico para o estudo aprofundado da Teoria dos Conjuntos. }\end{array}$ \\
\hline$S F \uparrow F$ & $\begin{array}{l}\text { V: transposição entre a zona de confluência dos mundos Simbólico e Formal e o Mundo } \\
\text { Formal. Desenvolve-se abstração operacional com vistas à abstração formal e, com ou } \\
\text { sem indícios de abstração estrutural. Ocorre a transição da matemática teórica para a }\end{array}$ \\
\hline
\end{tabular}




\begin{tabular}{|l|l|}
\hline & $\begin{array}{l}\text { matemática formal. Apresenta-se o Sistema Axiomático Formal para embasar a prova } \\
\text { matemática, ou seja, há aprimoramento do pensamento matemático ao transitar da } \\
\text { prova simbólica para a prova formal. }\end{array}$ \\
\hline $\begin{array}{l}\text { H: ações de matemática formal restritas ao Mundo Formal. A matemática Formal se } \\
\text { desenvolve, no âmbito da Geometria, por meio das definições conceptuais } \\
\text { transformando-as em geometrias axiomáticas que proporcionam as demonstrações e } \\
\text { formalidades (geometricamente), por exemplo: Método da Exaustão, Princípio de } \\
\text { Arquimedes, Teorema de Pitágoras, Teorema de Tales, Teorema Fundamental da } \\
\text { Semelhança, Teorema Fundamental da Proporcionalidade, Relação de Euler, com } \\
\text { destaque para os Elementos de Euclides e as proposições de Arquimedes. As } \\
\text { discussões oriundas da zona tripla de confluências entre os Três Mundos da } \\
\text { Matemática fomentam o desenvolvimento da matemática formal na construção das } \\
\text { provas formais com a Teoria dos Conjuntos e a Lógica Matemática, por exemplo: a } \\
\text { função matemática, a continuidade e descontinuidade de funções matemáticas, limites, } \\
\text { limites de funções matemáticas, derivadas, soma de Riemann, integrais definidas, } \\
\text { integrais indefinidas, o Teorema Fundamental da Aritmética, o Teorema Fundamental da } \\
\text { Álgebra e o Teorema Fundamental do Cálculo. } \\
\text { V: desenvolvimento de abstração formal. O aprimoramento do pensamento matemático } \\
\text { ocorre no processo de transformação das demonstrações e provas formais geométricas } \\
\text { e algébricas, em provas formais da Matemática por intermédio do Sistema Axiomático } \\
\text { Formal, no qual se destacam os Axiomas de Dedekind - Peano e a Álgebra Abstrata. }\end{array}$ \\
\hline
\end{tabular}

Fonte: Elaborado pelos autores com base em Polegatti (2020)

Polegatti (2020) ressalta que a dinâmica de movimento do pensamento matemático apresentada não é única, ela representa nosso olhar para uma amostra do desenvolvimento cognitivo do conhecimento matemático. Então, muito menos ela é completa, estanque, linear. O objetivo é conduzir a discussões e reflexões, além de apresentar-se uma amostra da possibilidade de abrangência do quadro teórico dos Três Mundos da Matemática, tanto nos momentos de planejamento de atividades, quanto nas análises e possíveis interpretações dessas atividades após suas aplicações.

A seguir, tem-se o caso hipotético com o cálculo das áreas de regiões limitadas por polígonos regulares, em função do raio da circunferência que os circunscreve e tendo como base, a fórmula matemática do cálculo da área da região limitada por um triângulo qualquer, a partir do valor do seno de um de seus ângulos internos. Planeja-se e analisam-se as discussões da atividade proposta na perspectiva do quadro teórico dos Três Mundos da Matemática.

\section{O CASO HIPOTÉtICO dO CÁlCULO DAS ÁREAS DE REGIÕES LIMITADAS POR POLÍGONOS REGULARES}

O cálculo das áreas de regiões limitadas por figuras planas faz parte do escopo da Geometria que está inserido no currículo de Matemática do Ensino Fundamental. Nesse 
contexto, o estudo envolvendo polígonos regulares prepara os estudantes para a compreensão do cálculo da área de regiões limitadas por círculos. Tall (2020) destaca que o estudo das definições dos objetos matemáticos que fazem parte de uma atividade matemática é fundamental para a construção de novos conceitos e manipulações matemáticas. De acordo com Moise \& Downs (1971), uma determinada região para ser classificada como polígono deve abranger três propriedades.

Seja $P_{1}, P_{2}, \ldots, P_{n}$, uma sequência de $n$ pontos distintos num plano com $n \geq 3$. Suponha que os $n$ segmentos $\overline{P_{1} P_{2}}, \overline{P_{2} P_{3}}, \ldots, \overline{P_{n-1} P_{n}}, \overline{P_{n} P_{1}}$ tem as seguintes propriedades: (1) Nenhum par de segmentos se intercepta a não ser nas suas extremidades; (2) Nenhum par de segmentos com extremidade comum é colinear. Então a reunião dos $n$ segmentos é chamada polígono (p. 473).

Para essa situação hipotética utiliza-se como exemplo dois polígonos regulares inscritos em circunferencias de raio medidindo $8 \mathrm{~cm}$. Um deles composto por cinco segmentos (pentágono) e o outro formado por oito segmentos (octógono). Segundo Moise \& Downs (1971), um polígono é classificado como regular se ele é convexo, se todos os seus lados e ângulos são congruentes entre si. "Um polígono é convexo se nenhum par de seus pontos está em semiplanos opostos relativamente a uma reta que contém um lado do polígono" (Moise \& Downs, 1971, p. 474).

Na Figura 2, apresenta-se o triângulo com seus lados $(a, b, c)$, sua altura $h$ seu ângulo $\alpha$ considerado, bem como os polígonos regulares (pentágono e octógono) utilizados na atividade proposta inscritos em circunferêcias de raio $r$, divididos em triângulos congruentes e demarcando os respectivos ângulos $\alpha$ originários dessas divisões. Ou seja, o valor do ângulo $\alpha$ depende da divisão de $360^{\circ}$ pelo número de lados $n$ de cada polígono, como descrito adiante em (4).

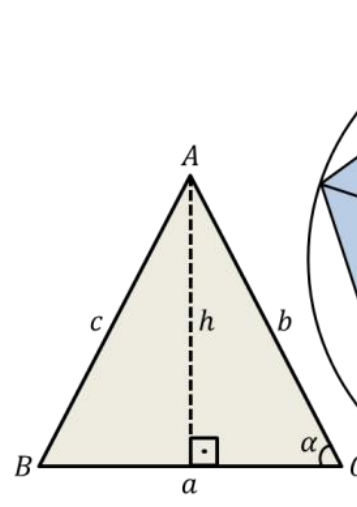

(I)

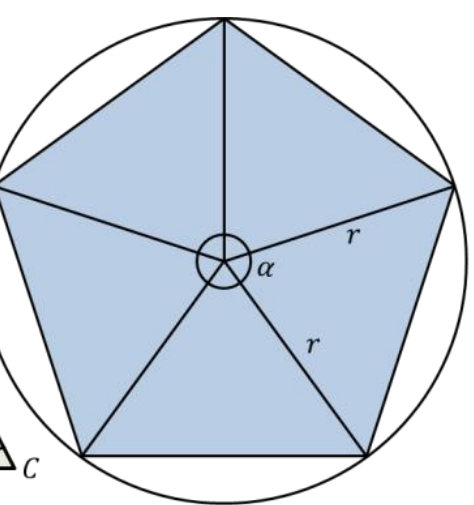

(II)

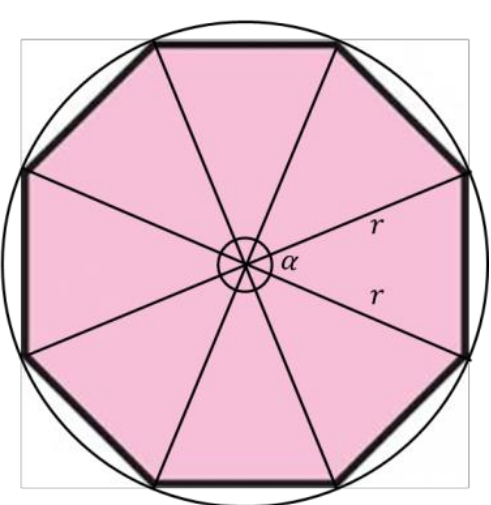

(III)

Figura 2: O triângulo, o pentágono e o octógono em destaque Fonte: Elaborada pelos autores 
Em meio às manipulações algébricas, o triângulo da imagem (I) é utilizado como base para a construção da fórmula (3) que serve para calcular a área da região limitada pelo mesmo, em função do valor do seno de um de seus ângulos internos (2), e das medidas dos lados $a$ e $b$ do referido triângulo que formam o ângulo $\alpha$ trabalhado. Parte-se da fórmula (1) que utiliza a base $a$ e a altura $h$ do triângulo da imagem (I) da Figura 2, para descrever o cálculo da área limitada pela região triangular $A_{T}$ em questão. Então, se

$$
A_{T}=\frac{a \cdot h}{2}
$$

$E$,

$$
\sin \alpha=\frac{h}{b} \rightarrow h=b \cdot \sin \alpha
$$

Tem-se que,

$$
A_{T}=\frac{a \cdot b \cdot \sin \alpha}{2}
$$

Seguindo com a atividade, promove-se discussões sobre a divisão dos polígonos regulares em triângulos congruentes como repesentado nas imagens (II) e (III) da Figura 2, no caso um pentágono e um octógono regulares, respectivamente. Percebe-se que a quantidade de triângulos obtidos e o valor do ângulo $\alpha$ dependem do número de lados $n$ de cada polígono regular.

O ângulo $\alpha$ pode ser determinado pela fórmula (4), e esta ao ser manipulada com a fórmula (3), na qual $a$ e $b$ são os lados do triângulo obtem-se a fórmula (5), na qual destaca-se que os lados $a$ e $b$ correspondem ao raio $r$ da circunferência que circunscreve cada polígono regular. Assim, por meio da fórmula (5), pode-se calcular a área da região limitada por qualquer polígono regular $A_{P r}$. Então, se

$$
\alpha=\frac{360^{\circ}}{n}
$$

Ao manipular-se algebricamente (3) e (4), obtem-se

$$
A_{P r}=\frac{r \cdot r \cdot \sin \left(\frac{360^{\circ}}{n}\right)}{2} \cdot n
$$

Perante o quadro teórico dos Três Mundos da Matemática, essas manipulações algébricas são articuladas e discutidas com os participantes da atividade no âmbito da zona de confluência entre o Mundo Simbólico e o Mundo Formal, região de cor verde na 
Figura 1. Em seus desdobramentos, aprimora-se o pensamento matemático por meio das ações de matemática teórica e ao desenvolver-se abstração operacional com indícios de abstração estrutural e com vistas a abstração formal. Com a finalidade de nortear-se as discussões da situação hipotética, apresenta-se na Figua 3 um panorama geral com os desdobramentos da atividade proposta, na pesrpectiva do quadro teórico dos Três Mundos da Matemática da Figura 1.

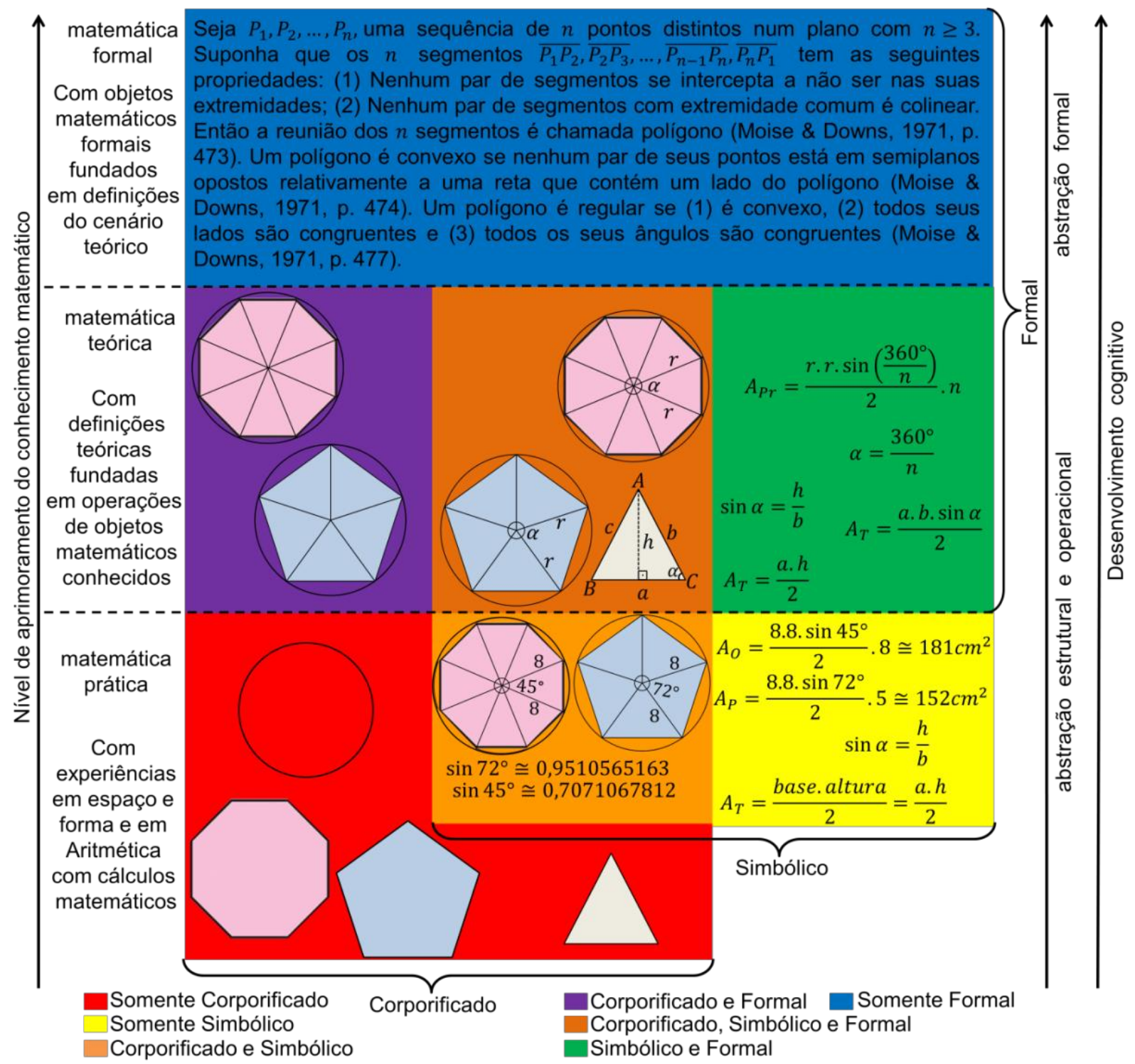

Figura 3: O cálculo da área de regiões limitadas por polígonos regulares em perspectiva no quadro teórico dos Três mundos da Matemática

Fonte: Elaborada pelos autores com base em Polegatti (2020)

No cenário da Figura 3, a atividade inicia-se com a dinâmica de movimento do pensamento matemático em ações de observação e manipulação em triângulos, pentágonos e octógonos regulares, desenvolvidas no campo da matemática prática e que 
transitam no âmbito do Mundo Corporificado. Para tanto, sugere-se a utilização de material rígido para a confecção desses entes geométricos. Tall (2020) salienta que no Mundo Corporificado as observações e manipulações dos objetos matemáticos partem da realidade para representações construídas na mente do ser humano. Nesse sentido, no transcorrer das manipulações deve-se ressaltar que os triângulos, pentágonos e octógonos regulares utilizados, assim como demais figuras planas bidimensionais, não são palpáveis, não existem no mundo real e sim no mundo das ideias matemáticas por meio de suas representações mentais construídas pelo indivíduo.

Após as discussões oriundas das observações e manipulações, a dinâmica de movimento do pensamento matemático transita entre o Mundo Corporificado e a zona de confluência dos mundos Corporificado e Formal, deslocando-se da matemática prática para a matemática teórica. Demarcam-se os centros no pentágono e octógono utilizados que correspondem aos centros das circunferências que os circunscreve, bem como se verifica que suas áreas equivalem à soma de cinco e oito áreas de regiões limitadas por triângulos, respectivamente.

A partir da zona de confluências dos mundos Corporificado e Formal adentra-se ao Mundo Formal transitando da matemática teórica para a matemática formal, dialogam-se as definições de polígono, polígono convexo e polígono regular. Já no âmbito da zona de confluência entre os mundos Corporificado e Simbólico, aprimora-se o pensamento matemático por meio das ações de matemática prática. Verifica-se no pentágono e no octógono: se possuem as duas propriedades previstas na definição de polígono, a condição para que sejam convexos e, após compreende-se que eles são polígonos convexos, com o auxílio de régua e transferidor aferem-se todos os lados e ângulos, respectivamente, para constatar se eles são congruentes (regulares).

Utiliza-se calculadora científica para obterem-se os valores aproximados dos senos dos ângulos de $45^{\circ}$ e $72^{\circ}$. De posse das medidas aferidas, realiza-se a transposição da zona de confluência dos mundos Corporificado e Simbólico para o Mundo Simbólico. Aprimora-se o pensamento matemático por meio de ações de matemática prática que envolvem os cálculos das áreas das regiões limitadas pelos polígonos regulares da atividade. Para tanto, manipula-se as fórmulas (1) e (2) para obter-se a fórmula (3). Ao final, multiplica-se o resultado de (3) pelo número de regiões triangulares que compõem cada polígono regular.

Por sua vez, no cenário da zona de tripla confluência dos Três Mundos da Matemática, por meio das ações de matemática teórica nos dois polígonos regulares em 
tela, aprimora-se o pensamento matemático ao compreender-se e generalizar-se (abstração operacional com indícios de abstração estrutural e com vistas à abstração formal), que os lados dos triângulos que compõem o seu ângulo central $\alpha$ correspondem ao raio $r$ da circunferência que circunscreve cada polígono regular.

Após essas discussões e reflexões, adentra-se à zona de confluência entre os mundos Simbólico e Formal. Nela, aprimora-se o pensamento matemático por meio de ações de matemática teórica com manipulações simbólicas que conduz a construção da fórmula (5). Esta, por conseguinte, serve para o cálculo da área de uma região limitada por um polígono regular qualquer, em função do raio da circunferência que o circunscreve e, tendo como base a área da região de um triângulo qualquer, a partir do valor do seno de um de seus ângulos internos.

\section{CONSIDERAÇÕES FINAIS}

De acordo com Martelozo \& Savioli (2019), o quadro teórico dos Três Mundos da Matemática se aplica tanto aos estudantes quanto aos professores que ensinam Matemática. Ele amplia os estudos e as pesquisas em Educação Matemática, serve como fonte de discussões e de como professores e pesquisadores lidam e debatem, entre si mesmos e com seus estudantes, o conhecimento matemático. No âmbito desse quadro teórico, articula-se o desenvolvimento do pensamento matemático por intermédio das ações humanas nos objetos matemáticos (matemática prática, matemática teórica e matemática formal), bem como por três formas de abstrair-se matematicamente (estrutural, operacional e formal). Neste caso, por exemplo, o que mais pode ser debatido a partir da fórmula (5)?

Em uma pesquisa sobre o estudo de limites para acadêmicos de uma Licenciatura em Matemática, Soares \& Cury (2017, p. 81), afirmam que o estudo de limites na perspectiva do quadro teórico dos Três Mundos da Matemática "favorece a compreensão dos conceitos e traz ideias para o trabalho na escola básica, em conteúdos que têm a noção de limite como pressuposto". Segundo Moise \& Downs (1971), conforme vai se aumentando o número de lados de um polígono regular, o valor da área da região limitada por um polígono com muitos lados aproxima-se da área da região limitada pelo círculo que o circunscreve. "A área de um círculo é o limite das áreas dos polígonos regulares inscritos" (1971, p. 484, grifo dos autores). 
Nesse sentido, com o apoio da calculadora científica do celular, que, geralmente, está sempre disponível, pode-se partir da fórmula (5) com o objetivo de construir-se a fórmula que se utiliza para o cálculo da área circular. Primeiro, considera-se o valor de $360^{\circ}$ para o ângulo $\alpha$, ou seja, um polígono regular com 360 lados. E então, a partir da fórmula (5) procede-se com o cálculo da expressão (6) e, na Figura 4 apresenta-se a imagem o resultado de sua parte numérica na calculadora do celular.

$$
\frac{\text { r.r. } \sin \left(\frac{360^{\circ}}{360}\right) \cdot 360}{2} \cong r^{2} \cdot \pi
$$

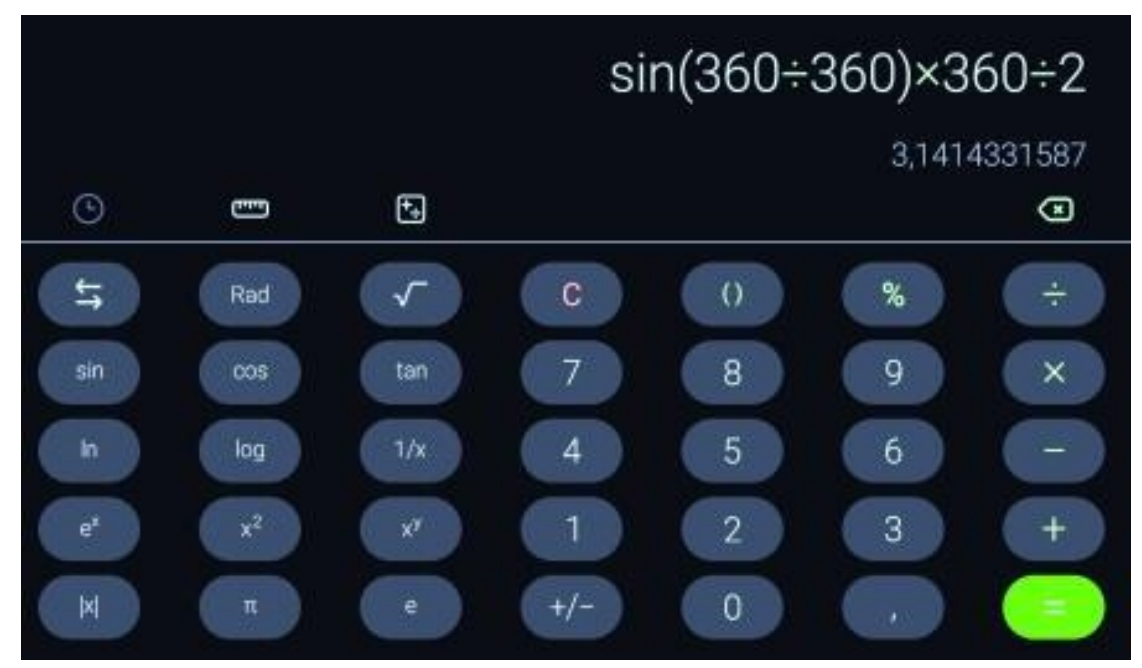

Figura 4: $O$ resultado do cálculo da expressão (6) para $n=360$ no visor da calculadora Fonte: Elaborada pelos autores

Verifica-se que o resultado desse cálculo para um polígono regular de 360 lados corresponde ao valor aproximado, com duas casas decimais, que utilizamos para $\pi$ na Educação Básica $(3,14)$. Ou seja, assim como afirmado por Moise \& Downs (1971), o valor da área da região limitada por um polígono regular de 360 lados é aproximado do valor da área da região limitada pelo círculo que circunscreve o referido polígono regular. Indo além, qual o valor aproximado de $\pi$ que a calculadora do celular utilizada descreve? E ao considerar-se o limite da quantidade de números suportado para a calculadora em questão, qual é o resultado da área da região limitada por esse polígono regular?

Salienta-se que essa calculadora tem um limite para a quantidade de números estabelecida em 15 dígitos. Assim, ao pensar-se no maior valor possível para o número de lados do polígono regular utiliza-se quinze vezes o número nove, quase um quatrilhão de lados. Na Figura 5, em sua imagem (I) apresenta-se o valor aproximado de $\pi$ adotado 
por essa calculadora, bem como em sua imagem (II) o visor da calculadora com o resultado da operação para um polígono regular com o valor máximo de lados suportado pela referida calculadora.

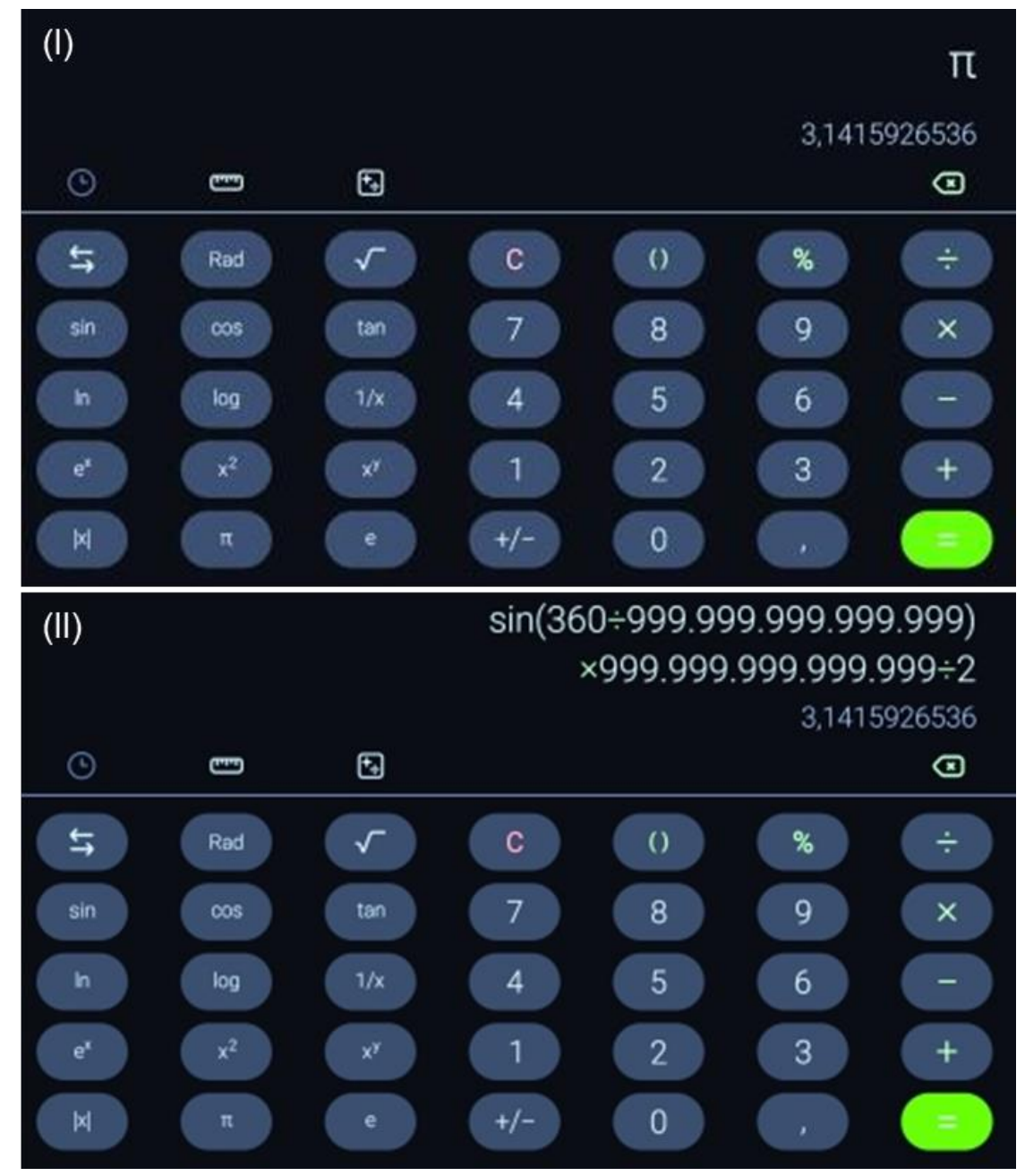

Figura 5: O valor aproximado de $\pi$ e o resultado da parte numérica para o cálculo da área da região limitada por um polígono regular com o número máximo de lados suportado pela calculadora Fonte: Elaborada pelos autores

Verifica-se que os valores obtidos coincidem, ou seja, o valor aproximado de $\pi$ pela calculadora $(3,1415926536)$, corresponde à parte numérica da fórmula (5) quando se adota para o número de lados $n$ do polígono regular o valor máximo que a calculadora suporta. Nesse sentido, a partir da fórmula (5) pode-se considerar que quando o limite do número de lados $n$ do polígono regular circunscrito tende ao infinito ( $\infty)$, a área da região limitada por esse polígono regular se aproxima da área da região limitada pelo círculo que o circunscreve, como descrito em (7). 


$$
\lim _{n \rightarrow \infty}\left[\frac{r^{2} \cdot \sin \left(\frac{360^{\circ}}{n}\right)}{2} \cdot n\right]=\pi \cdot r^{2}
$$

Por fim, conclui-se que os estudos, planejamentos, execuções e as análises de processos de ensino e aprendizagem da Matemática por meio do quadro teórico dos Três Mundos da Matemática, como apontam Martelozo (2019), Polegatti (2020) e Flôres (2020), conduzem a reflexões sobre o que foi planejado e o que mais pode ser dialogado na atividade que se está aplicando. Ou seja, a versatilidade, abrangência e dinâmica desse referido quadro teórico possibilitam ao professor, vislumbrar para além do que inicialmente está proposto em processos de ensino e de aprendizagem da Matemática, apresentando-se como essencial o seu estudo na formação inicial e continuada de professores que ensinam Matemática.

\section{REFERÊNCIAS}

Bisognin, E., Bisognin, V. \& Leivas, J. C. P. (2016). Aprendizagem de sequências numéricas: pesquisa sobre dificuldades de licenciandos em Matemática. Zetetiké, 24(3), 361-377. doi: https://doi.org/10.20396/zet.v24i3.8648090

Bueno, R. W. S. \& Viali, L. (2019). O Cálculo e os Três Mundos da Matemática: um estado do conhecimento. DYNAMIS, 25(2), 39-55. doi: http://dx.doi.org/10.7867/1982$\underline{4866.2019 v 25 \mathrm{n} 2 \mathrm{p} 39-55}$

Flôres, M. V. (2020). Construção dos números racionais na licenciatura: um estudo desenvolvido à luz dos Três Mundos da Matemática (Tese de Doutorado em Ensino de Ciências e Matemática) Universidade Franciscana, Santa Maria.

Lima, R. N. (2019). Dispositivos móveis em sala de aula: uma jornada pelos Três Mundos da Matemática. REVEMAT, 14(1), 1-21. doi: https://doi.org/10.5007/1981$\underline{1322.2019 . e 59790}$

Martelozo, D. P. S. \& Savioli, A. M. P. D. (2019). Já-encontrados na aprendizagem da Matemática: quais implicações? VIDYA, 39(1), 55-71. in: https://periodicos.ufn.edu.br/index.php/VIDYA/article/view/2622

Martelozo, D. P. S. (2019). Interações entre cognição e afetividade na aprendizagem da Matemática (Tese de Doutorado em Ensino de Ciências e Educação Matemática) Universidade Estadual de Londrina, Londrina.

Moise, E. E. \& Downs, F. L. (1971). Geometria moderna. Edgard Blücher, São Paulo.

Polegatti, G. A. (2020). Jornadas pelos Três Mundos da Matemática sob perspectiva do Programa Etnomatemática na Licenciatura Intercultural Indígena (Tese de Doutorado 
em Ensino de Ciências e Educação Matemática) Universidade Estadual de Londrina, Londrina.

Schastai, M. B. (2017). Tall e Educação Matemática Realística: algumas aproximações (Tese de Doutorado em Ensino de Ciências e Educação Matemática) Universidade Estadual de Londrina, Londrina.

Soares, G. O. \& Cury, H. N. (2017). O conteúdo de limite em cursos de Licenciatura em Matemática: uma pesquisa à luz dos Três Mundos da Matemática. ReBECEM, 1(1), 64-83. doi: https://doi.org/10.33238/ReBECEM.2017.v.1.n.1.18557

Tall, D. O. (2007). Embodiment, Symbolism and Formalism in Undergraduate Mathematics Education. In Proceedings 10th Conference of the Special Interest Group of the Mathematical Association of America on Research in Undergraduate Mathematics Education. (pp. 1-18). San Diego, Califórnia, USA. In: http://homepages.warwick.ac.uk/staff/David.Tall//pdfs/dot2007b-rume-keynote.pdf

Tall, D. O. (2008). The Transition to Formal Thinking in Mathematics. Mathematics Education Research Journal, p. 1n: http://homepages.warwick.ac.uk/staff/David.Tall/pdfs/dot2008e-meri-3worlds.pdf

Tall, D. O. (2013). How Humans Learn to Think Mathematically: Exploring the three worlds of mathematics. Cambridge: Cambridge University Press.

Tall, D. O. (2019). Complementing supportive and problematic aspects of mathematics to resolve transgressions in long-term sense making. In Proceedings Fourth Interdisciplinary Scientific Conference on Mathematical Transgressions, Cracow, Poland: Institute of Mathematics of the Pedagogical University of Cracow. (pp. 1-27). In: $\quad$ http://homepages.warwick.ac.uk/staff/David.Tall/pdfs/dot2019a-transgressionskrakow.pdf

Tall, D. O. (2020). Making Sense of Mathematical Thinking over the Long Term: The Framework of Three Worlds of Mathematics and New Developments. In D. O. Tall, \& I. Witzke, (Eds.), MINTUS: Beiträge zur mathematischen, naturwissenschaftlichen und technischen Bildung. (pp. 1-26). Wiesbaden: Springer. In: http://homepages.warwick.ac.uk/staff/David.Tall/pdfs/dot2020a-3worlds-extension.pdf

Visintainer, M. (2019). Significados de Cônicas à luz dos Três Mundos da Matemática. (Tese de Doutorado em Educação Matemática) Universidade Anhanguera de São Paulo, São Paulo. 


\section{NOTAS}

\section{TÍTULO DA OBRA}

Os Três Mundos da Matemática na formação de professores que ensinam Matemática: o cálculo da área limitada por regiões poligonais regulares.

\section{Geraldo Aparecido Polegatti}

Doutor em Ensino de Ciências e Educação Matemática

Professor do Ensino Básico, Técnico e Tecnológico, D4 Nível 1

Instituto Federal de Mato Grosso, Campus Juína, Juína, Mato Grosso, Brasil.

geappolegatti@gmail.com

Ohttps://orcid.org/0000-0003-4515-3855

Angela Marta Pereira das Dores Savioli

Doutora em Matemática

Professora Associada

Universidade Estadual de Londrina, Departamento de Matemática, Londrina, Paraná, Brasil.

angelamarta@uel.br

Ohttps://orcid.org/0000-0002-5624-6398

Endereço de correspondência do principal autor

Rua Evaldo Bento Vieira, 252, Residencial Rubi, casa 10, Fontana 1, Porto Seguro, Bahia, CEP:45810-000

\section{AGRADECIMENTOS}

Não se aplica.

\section{CONTRIBUIÇÃO DE AUTORIA}

Concepção e elaboração do manuscrito: G. A. Polegatti, A. M. P. D. Savioli

Coleta de dados: G. A. Polegatti, A. M. P. D. Savioli

Análise de dados: G. A. Polegatti, A. M. P. D. Savioli

Discussão dos resultados: G. A. Polegatti, A. M. P. D. Savioli

Revisão e aprovação: G. A. Polegatti, A. M. P. D. Savioli

\section{CONJUNTO DE DADOS DE PESQUISA}

Todo o conjunto de dados que dá suporte aos resultados deste estudo foi publicado no próprio artigo.

\section{FINANCIAMENTO}

Esse trabalho tem auxílio financeiro de forma indireta pela Coordenação Aperfeiçoamento de Pessoal de Nível Superior (CAPES).

\section{CONSENTIMENTO DE USO DE IMAGEM}

Não se aplica.

\section{APROVAÇÃO DE COMITÊ DE ÉTICA EM PESQUISA \\ Não se aplica.}

\section{CONFLITO DE INTERESSES}

Não se aplica.

LICENÇA DE USO - uso exclusivo da revista

Os autores cedem à Revemat os direitos exclusivos de primeira publicação, com o trabalho simultaneamente licenciado sob a Licença Creative Commons Attribution (CC BY) 4.0 International. Estra licença permite que terceiros remixem, adaptem e criem a partir do trabalho publicado, atribuindo o devido crédito de autoria e publicação inicial neste periódico. Os autores têm autorização para assumir contratos adicionais separadamente, para distribuição não exclusiva da versão do trabalho publicada neste periódico (ex.: publicar em repositório institucional, em site pessoal, publicar uma tradução, ou como capítulo de livro), com reconhecimento de autoria e publicação inicial neste periódico.

PUBLISHER - uso exclusivo da revista

Universidade Federal de Santa Catarina. Grupo de Pesquisa em Epistemologia e Ensino de Matemática (GPEEM). Publicação no Portal de Periódicos UFSC. As ideias expressadas neste artigo são de responsabilidade de seus autores, não representando, necessariamente, a opinião dos editores ou da universidade.

EDITOR - uso exclusivo da revista

Méricles Thadeu Moretti e Rosilene Beatriz Machado

HISTÓRICO - uso exclusivo da revista

Recebido em: 06-07-2021 - Aprovado em: 13-10-2021 